$10^{\text {th }}$ Winter Workshop on Nuclear Dynamics, Snowbird, Utah(Jan. 15-21, 1994)

\title{
Pion Interferometry in E814 - Toward Equilibrium at the AGS
}

\author{
$\mathrm{Nu} \mathrm{Xu}$ for the E814 Collaboration ${ }^{1}$ \\ Department of Physics \\ State University of New York at Stony Brook \\ Stony Brook, New York 11794-3800
}

\begin{abstract}
We report recent pion interferometry results from AGS experiment E814. By comparing to the results of RQMD calculations, a freeze-out size $R_{r m s}=8.3 \mathrm{fm}$ is found in $\mathrm{Si}+\mathrm{Pb}$ central collisions. A consistent thermal equilibration picture is established by comparing experimental data with the results of both hydrodynamic and cascade model calculations.
\end{abstract}

\section{Introduction}

The ultimate goal of ultra-relativistic heavy-ion program at the BNL AGS and CERN SPS is to study highly excited nuclear matter and the transition from hadronic matter to quark gluon plasma. In the QGP state, quarks and gluons are no longer confined but they move freely in a fairly large volume. According to our knowledge, the de-confined state can be approached via either compression or heating of nuclear matter in heavy-ion collisions.

At AGS energies, the most relevant process is believed to be compression. However, before we try to identify any exotic events such as, for example QGP phase transition or chiral symmetry restoration, we have to answer the following questions: (1) Were the densities(due to the compression) high enough? (2) Was the high density region large enough? (3) Was the system thermalized?

The first question has been addressed in depth in reference [1], where the main conclusion is: a high degree of stopping has been reached in the $\mathrm{Si}+\mathrm{Pb}$ central collisions. The baryon density is as high as $5 \rho_{0}$ over a period of about $5 \mathrm{fm} / \mathrm{c}[2]$. In order to address questions (2) and (3), we will organize the paper as follows: in section 2, we will discuss recent pion interferometry results from the E814 collaboration. The pion source size as a function of time will also be studied. In section 3, we will discuss the thermal equilibration issue at AGS energies using two different approaches, namely, a hydrodynamical type calculation and a cascade model, RQMD [2], calculation. Finally, we will make some concluding remarks.

\footnotetext{
${ }^{1}$ BNL, GSI, McGill University, University of Pittsburgh, SUNY Stony Brook, University of Sao Paulo, Wayne State University, Yale University
} 


\section{Recent HBT results from E814}

For the data presented here we used the E814 apparatus which provides a $4 \pi$ event characterization with a target calorimeter and a charged particle multiplicity detector. The forward spectrometer acceptance was determined by a lead collimator to $-115<\theta_{\mathbf{x}}<14 \mathrm{mr}$ (bending plane) and $-21<\theta_{\mathbf{y}}<21 \mathrm{mr}$, both with respect to the beam direction. Particle identification is obtained by combination of time-of-flight and momentum measurement [1]. The acceptance in transverse momentum $p_{t}$ and rapidity $y$ of identified $\pi^{+}$and $\pi^{-}$is shown in Fig. 1. Note that pions of both charges are detected at $p_{t} \geq 0$ with a mean rapidity of about 3 .

Experimentally the two-particle correlation function $\mathrm{C}_{2}$ is defined as:

$$
C_{2}(q)=\frac{N_{t r}(q)}{N_{b k}(q)}
$$

where $q=\sqrt{-\left(p_{1}-p_{2}\right)^{2}}$ is the relative 4-momentum between the two identical particles. As done frequently, the numerator $N_{t r}(q)$ is obtained by taking two particles from the same event, while the denominator $N_{k k}(q)$ is constructed using two pions from different events (mixed event technique). This way one ensures that the statistical errors in the correlation function are determined by the statistics of the true pion pairs only.

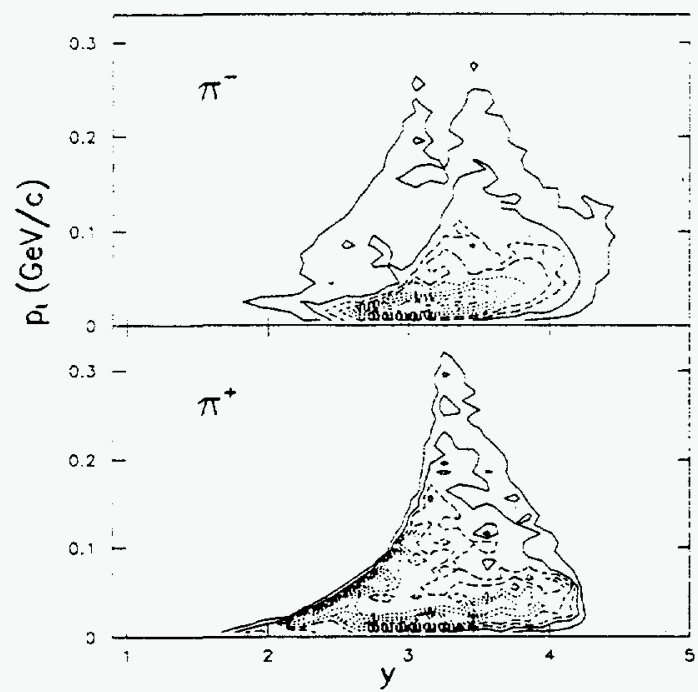

Figure 1. E814 acceptance.

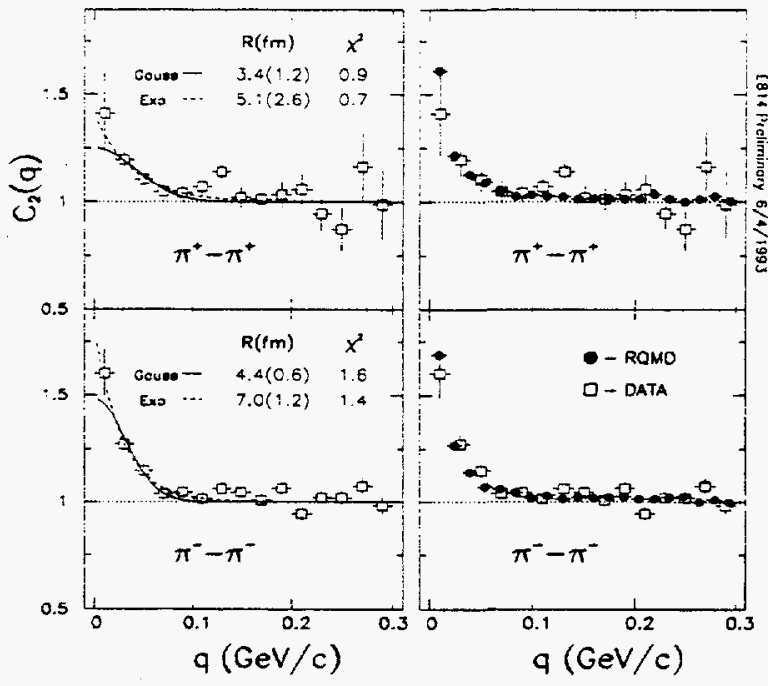

Figure 2. Correlation functions: (left) measured data with Gaussian and exponential fits; (right) data with RQMD results.

The pion correlation functions corrected for Coulomb effects and two-particle acceptance are shown in Fig. 2 (open symbols). The total number of selected $\pi^{-}\left(\pi^{+}\right)$ pairs is $23.4 \mathrm{k}(4.3 \mathrm{k})$ and about $80 \%$ of the pairs are in the relative momentum range of $0 \leq q \leq 0.3 \mathrm{GeV} / \mathrm{c}$. Error bars are statistical only. The background distribution 


\section{DISCLAIMER}

Portions of this document may be illegible in electronic image products. Images are produced from the best available original document. 
is normalized to the total number of the entries in the true distributions within the range of $0 \leq \mathrm{q} \leq 1 \mathrm{GeV} / \mathrm{c}$. It can be seen from this figure that, for $\mathrm{q} \geq 0.1 \mathrm{GeV} / \mathrm{c}$, the distribution is consistent with unity. The Bose-Einstein enhancement is clearly visible in the low relative momentum region for both like-sign pion pairs.

The measured correlation functions have been fitted by two commonly used parameterization, namely, a Gaussian and an exponential function:

$$
C_{2}^{g}(q)=1+\lambda_{g} \cdot \exp \left(-q^{2} R_{g}^{2}\right) ; \quad C_{2}^{e}(q)=1+\lambda_{e} \cdot \exp \left(-q R_{e}\right) .
$$

where $\lambda$ is the chaoticity parameter and $R$ is determined by the space-time extent of the pion source. In Fig. 2 (left), the solid and dashed lines represent the Gaussian and exponential fits, respectively. The extracted fit parameters are also summarized in the figure. While the reduced $\chi^{2}$ is slightly smaller for the exponential fit, both functional forms are consistent with the data. However, the source parameters are quite different.

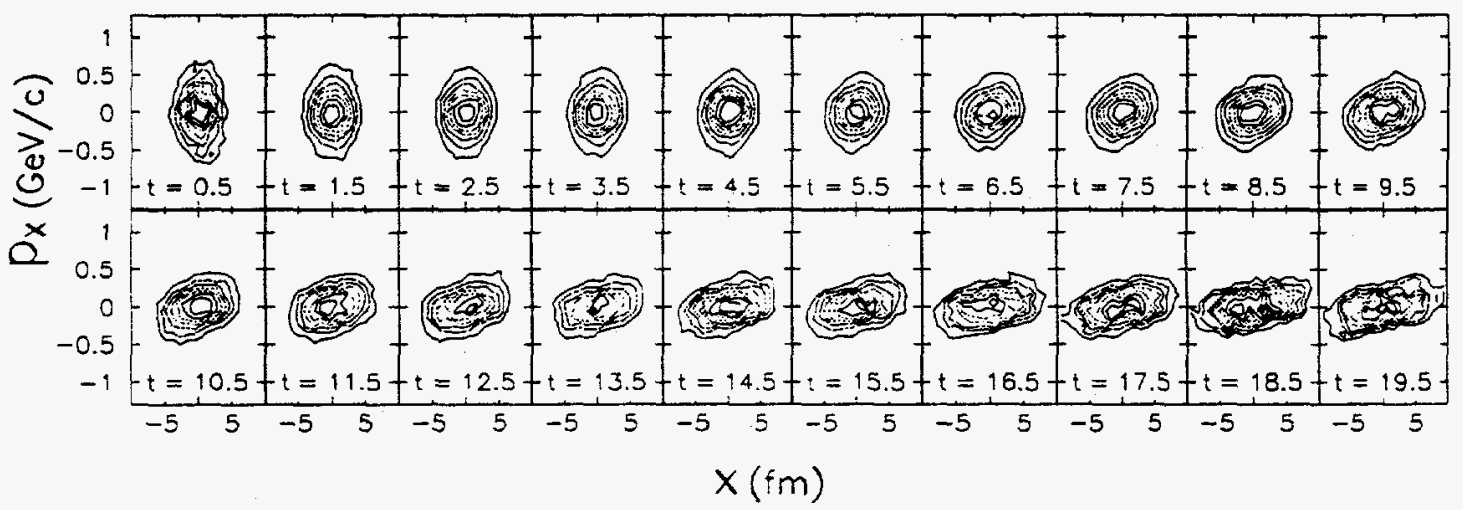

Figure 3. Contours of RQMD pion phase-space distribution for central Si+ $\mathrm{Pb}$ collisions. Each window represents a time interval of $\Delta t_{c m}=1.0 \mathrm{fm} / \mathrm{c}$.

Non-Gaussian shapes of the pion correlation function have been observed in heavyion collisions as well as in hadron-hadron collisions $[3,4,5]$. The shape of correlation function depends, besides the source distribution, on the experimental acceptance, resonance decays, dynamics of the emitting source, and other effects[6]. To overcome these ambiguities, rather than extracting the source size from fitting a certain functional form to the data, we use dynamical models with a known space-time characteristics of the source, impose the Bose-Einstein effect and evaluate the two particle correlation functions to compare them with the experimental results.

The event generator RQMD [2] has been successful in describing many aspects of pion, proton, kaon, and other measured single-particle spectra $[1,7,8,9]$ at both AGS and SPS energies. It is natural to use the model to also calculate the two-particle correlation functions.

Pion phase-space distributions from RQMD for a dimension $x$ transverse to the beam direction are shown in Fig. 3 for $14.6 \mathrm{~A} \cdot \mathrm{GeV} / \mathrm{c}^{28} \mathrm{Si}+\mathrm{Pb}$ collisions (for $b \leq 1.0$ 
$\mathrm{fm})$. These distributions were generated for pions at different times in the collision. As shown in Fig. 3, as time elapses, (1) the spatial distributions become wider implying that the source expands; and (2) a correlation between momentum and spatial coordinates also developes. An immediate consequence of the momentumspatial correlation is that a spectrometer will not be able to 'see' particles emitted from all locations of the source. Hence the source size extracted by fitting a functional form to the measured $\mathrm{C}_{2}$ will be reduced. Indeed, with a coverage $p_{t} \geq 150 \mathrm{MeV} / \mathrm{c}$, our $\pi^{+}$correlation functions [10] have shown a smaller size parameter $R_{g}=2.2 \mathrm{fm}$.

In order to generate a two particle correlation function, the Koonin-Pratt method [11] was used to construct a symmetrized pion wave function from the RQMD generated single-particle distributions. The experimental conditions were imposed on the RQMD event before feeding them into the calculation. Finally the correlation function was corrected by the Gamow factor as has been done for the experimental data. Results of the calculations, for both $\pi^{+}$and $\pi^{-}$within the E814 spectrometer, are shown in Fig. 2 (right) as filled circles. The agreement between the experiment and the model is excellent. Inspecting the RQMD phase-space distribution at freeze-out stage, we obtain a source size of $R_{r m s}=8.3 \mathrm{fm}$ (in a frame with $\mathrm{y}_{\mathrm{cm}} \approx 1.3$ ).

\section{Thermal equilibrium at the $\mathbf{A G S ^ { 2 }}$}

Using a hydrodynamical model one can calculate the bulk properties of the relativistic heavy-ion collisions which will provide a macroscopic description of such collisions. On the other hand, a cascade type calculation which is basically the superposition of elementary collisions including resonance and mean-field effects provides a microscopic picture of the collisions. However, in the limit of thermal equilibrium, these two approaches should converge. It is important to realize that the thermal characteristics in the measured hadron spectra provides a necessary[12], but not sufficient, proof of thermal equilibration at the early stage of the collision.

\subsection{The hydrodynamical approach}

Let us start with a isotropic stationary thermal source:

$$
E \frac{d^{3} N}{d \vec{p}} \propto E e^{-E / T} \propto m_{t} \cosh (y) e^{-m_{t} \cosh (y) / T}
$$

where $m_{t}=\sqrt{p_{t}^{2}+m^{2}}$ and $T$ is the Boltzmann temperature. All kinematic variables are in the nucleon-nucleon center of mass system.

Integrating over the transverse variable $m_{t}$, one obtains the rapidity distribution:

$$
\frac{d N^{\prime}}{d y} \propto m^{2} T\left(1+2 \chi+2 \chi^{2}\right) e^{-1 / x}
$$

\footnotetext{
${ }^{2}$ In collaboration with P. Braun-Munzinger and J. Stachel
} 
with the parameter $\chi=T /(m \cosh (y))$. It is clear that the distribution is rather sensitive to the ratio of $m / T$. While the distribution for pions is close to that for massless particles, protons from an isotropic source are much narrower.

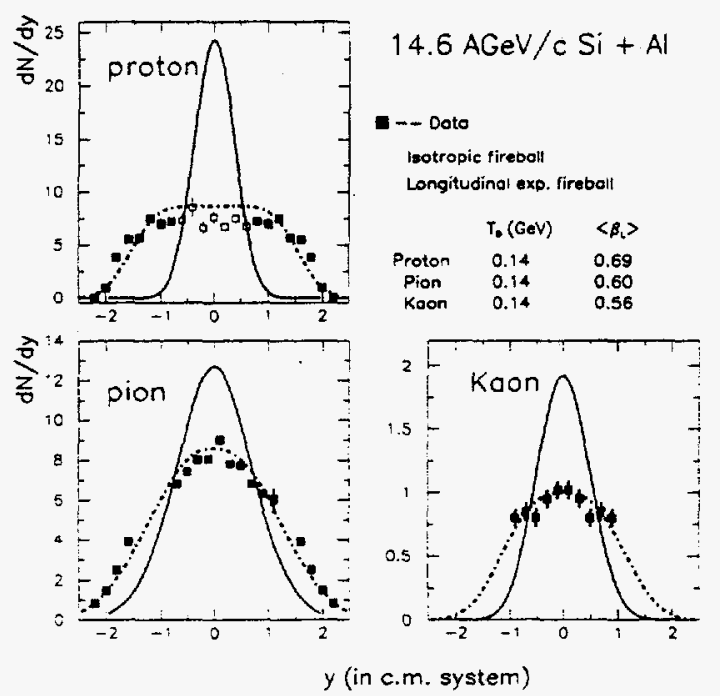

Figure 4. Rapidity distributions for 14.6A. GeV/c Si+Al collisions. Data are taken from E814 and E802 [1,13].
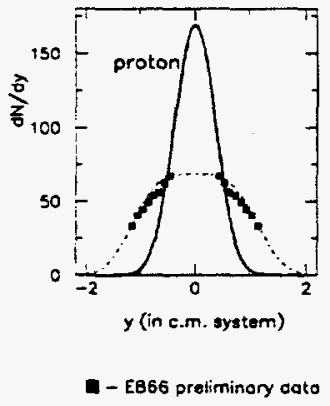

$T_{B}(G \circ V)\left\langle B_{1}\right\rangle$

Proton $\quad 0.14 \quad 0.56$

$\begin{array}{lll}K^{*}, K . \pi^{*} & 0.14 \quad 0.52\end{array}$

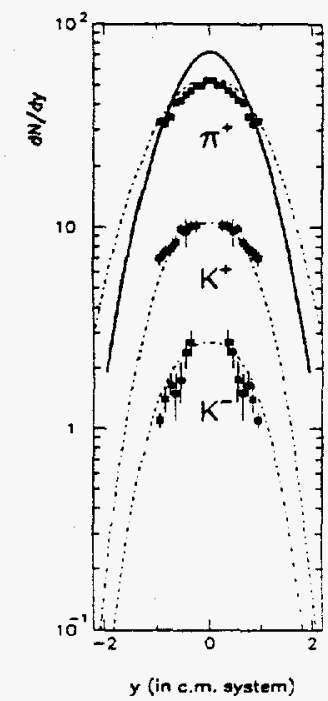

Figure 5. Rapidity distributions for $11.6 \mathrm{~A} \cdot \mathrm{GeV} / \mathrm{c} \mathrm{Au}+\mathrm{Au}$ collisions. Data are from $E 866$ [14].

Figs. 4 and 5 represent rapidity distributions for $14.6 \mathrm{~A} \cdot \mathrm{GeV} / \mathrm{c} \mathrm{Si}+\mathrm{Al}$ and 11.6A.GeV/c Au + Au central collisions, respectively. Experimental data are shown as square symbols and the isotropic thermal calculations (for $T=0.14 \mathrm{GeV}$ ) are shown as solid lines. Obviously the agreement between the calculation and data is poor. From previous studies $[1,2]$ we learned that a high degree of stopping has been reached at AGS energies and a density gradient is building up in the collision zone. Such collision induced pressure will eventually lead to collective flow. In order to include such effects into our model, following $[15,16]$, the final rapidity distribution is calculated by superposition of individual isotropic thermal sources within a rapidity interval $\left[-\eta_{\max }, \eta_{\max }\right]$ :

$$
\frac{d N}{d y}=\int_{-\eta_{\max }}^{\eta_{\max }} d \eta \frac{d N^{\prime}(y-\eta)}{d y} .
$$

Here the integration limit $\eta_{\max }$ is treated as a fit parameter. The results of a calculation for a longitudinal expanded fireball are shown as dash-dotted lines in Figs. 4 and 5 and the agreement with the data is excellent. While the extracted mean longitudinal velocity $\beta_{z}$ for protons is higher than that of the produced particles (pions and kaons) for the $\mathrm{Si}+\mathrm{Al}$ collisions, the extracted values for protons, pions

\footnotetext{
${ }^{3}$ The mean value of the $\beta_{l}$ is defined as: $\beta_{l}=\tanh \left(\eta_{l}\right)$ with $\eta_{l}=\eta_{\max } / \sqrt{3}$.
} 
and kaons are found to be rather close to each other for the heavy collision system $\mathrm{Au}+\mathrm{Au}$. Similar agreement is also obtained for the transverse spectra in $\mathrm{Si}+$ Au collisions (Fig. 6) where a common freeze-out temperature and the maximum transverse velocity are found to be $T=0.14-0.15 \mathrm{GeV}$ and $\beta_{t}^{\max } \approx 0.5$, respectively. A velocity profile $\beta_{t}=\beta_{t}^{\max } \cdot(r / R)^{2}(R=7.0 \mathrm{fm})$ has been used in the calculation[15].

\subsection{The cascade approach}

As we already mentioned, in the limit of thermal equilibration, a cascade type calculation should also show hydrodynamic effects. In the following we will discuss mean transverse outward ${ }^{4}$ velocity $\beta_{\text {out }}$ and longitudinal velocity $\beta_{z}$ from RQMD events. It is important to perform such tests since they can demonstrate, that even starting from purely binary collisions, thermal equilibrium and collective motion can be reached in heavy-ion collisions at AGS energies.

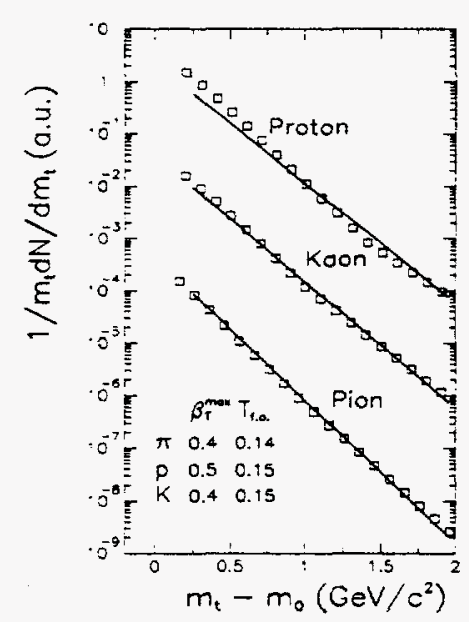

Figure 6. $m_{t}$ spectra (E802 [13]) for central Si + Au collisions. Thermal calculations are shown as open symbols.

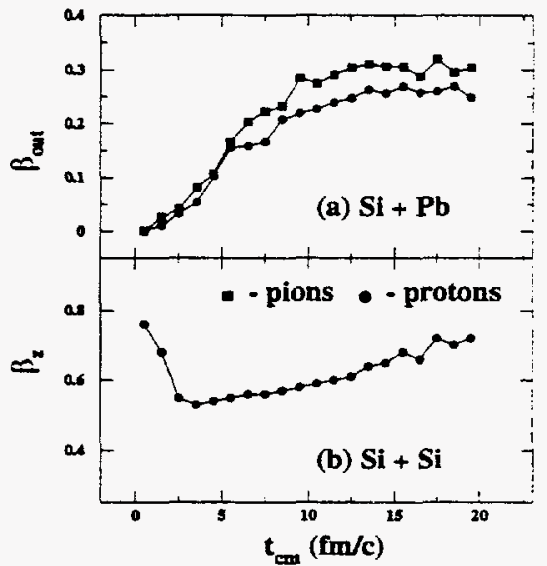

Figure 7. RQMD (a) transverse outward, (b) longitudinal velocity distributions for central collisions.

Fig. 7 (a) shows the mean outward velocity $\beta_{\text {out }}$ in $14.6 \mathrm{~A} \cdot \mathrm{GeV} / \mathrm{c} \mathrm{Si}+\mathrm{Pb}$ central collisions. Firstly we find a rapid increase in $\beta_{\text {out }}$ for the first $6 \mathrm{fm} / \mathrm{c}$, indicating a strong push from the high density region; secondly, when the acceleration is large, pions and protons share a similar velocity, implying transverse matter flow. This behaviour is indeed what one would expect from hydrodynamics; thirdly, for $t_{c m}>10$ $\mathrm{fm} / \mathrm{c}$, pions and protons are separated slightly and $\beta_{\text {out }}$ reaches a value of about 0.3 and 0.25 for pions and protons, respectively.

In the NN c.m. system, mean longitudinal velocities $\beta_{z}$ are calculated for $\mathrm{Si}+\mathrm{Si}$ central collisions at $14.6 \mathrm{~A} \cdot \mathrm{GeV} / \mathrm{c}$ (Fig. 7 (b)). There are clearly two distinct regions:

\footnotetext{
${ }^{4} \beta_{\text {out }}$ is the mean outward velocity: $\beta_{\text {out }}=\vec{\beta}_{t} \cdot \overrightarrow{r_{t}}$. In this case, $\beta_{\text {out }}$ is the only relevant variable in order to compare to hydrodynamic calculations.
} 
for $t_{c m} \leq 4 \mathrm{fm} / \mathrm{c}$, when the two incoming nuclei collide violently, the system is in a process of compression and $\beta_{z}$ decreases. At $\mathrm{t}_{\mathrm{cm}} \approx 4 \mathrm{fm} / \mathrm{c}, \beta_{z}$ reaches its minimum value of 0.5 . After this point the system experiences expansion and $\beta_{z}$ gradually approaches 0.7 . The initial decrease and later on rise of the flow velocity was also predicted [17] by Brown et al. in a thermal model calculation. Remember that if a transparency scenario was suited for such collisions, no increase of $\beta_{z}$ should occur.

\section{Conclusions}

We demonstrated, from analysing two-pion correlation functions, that the pion freeze-out source is large. The RMS value of the size is consistent with $R_{r m s}=$ $8.3 \mathrm{fm}$ in central $\mathrm{Si}+\mathrm{Pb}$ collisions. We also demonstrated, from comparing the experimentally measured spectra with the results of thermal and cascade calculations, that the thermal equilibrium scenario provides a consistence picture for collisions at the AGS energies. The large final stage source size is, in this picture, due to collective flow which expands the system from an initial transverse size $R_{T}=2.5 \mathrm{fm}$ to freeze-out size of $R_{T}=6.7 \mathrm{fm}$. Taking the maximum transverse velocity as 0.5 and assuming a linear dependence of the flow velocity on the radius, this implies a minimum expansion time of $t \geq 15 \mathrm{fm} / \mathrm{c}$.

\section{Acknowledgements}

The author is indebted to Drs. S. Pratt and H. Sorge for valuable discussions and their kindness to provide computer codes. We would also like to thank Drs. G. Brown, V. Koch, Y. Pang, and E.V. Shuryak for exciting and valuable discussions. We are grateful for support received from the U.S. DOE, U.S. NSF, Canadian NSERC, and CNPq Brazil.

\section{References}

[1] J. Barrette, et al., E814 Coll., Z. Phys., C59, 211(1993).

[2] H. Sorge, A. von Keitz, R. Mattiello, H. Stöcker, and W. Greiner, Phys. Lett. B243, 7(1990) and H. Sorge, R. Mattiello, H.Stöcker, and W. Greiner, Phys. Lett. B271, $37(1991)$.

[3] A. Bamberger, et al., NA35 Coll., Phys. Lett. B203, 320(1988).

4 T. Âkesson, et al., AFS Coll., Z. Phys. C36, 517(1987).

5 R. Albrecht, et al., Z. Phys. C53, 225(1992).

6. W.A. Zajc, in "Particle Production in Highly Excited Matter", H.Gutbrod and J.Rafelski, eds., (Plenum, New York,1993)p435.

[7] T. Hemmick, E814 Coll., Nucl. Phys. A566, 435c(1994).

8. J.P. Sullivan, et al., Phys. Rev. Lett., 70, 3000(1993).

9. Th. Schönfeld, et al., Nucl. Phys. A544, 439c(1991).

[10] N. Xu E814 Coll., Proceedings of HIPAGS'93, Workshop, MIT LNS-2158.

11 S. Pratt, Phys. Rev. Lett. 53, 1219(1984); S. Pratt, Phys. Rev. D33, 72(1986).

[12] E.V. Shuryak, Phys. Lett. B42, 357(1972); E.V. Shuryak, "The QCD Vacuum, Hadrons and the Superdense Matter", (World Scientific, Singapore, 1988).

[13] C. Parsons E802 Coll., Proceedings of HIPAGS'93, Workshop, MIT LNS-2158.

14. M. Gonin E802/E866 Coll., Proceedings of HIPAGS'93, Workshop, MIT LNS-2158.

15. E. Schnedermann, J. Sollfrank, and U. Heinz, Phys. Rev. C48, 2462(1993).

16 A.N. Makhlin, private communication.

[17] G. Brown, C.M. Ko, Z.G. Wu, and L.H. Xia, Phys. Rev. C43, 1881(1991). 


\section{DISCLAIMER}

This report was prepared as an account of work sponsored by an agency of the United States Government. Neither the United States Government nor any agency thereof, nor any of their employees, makes any warranty, express or implied, or assumes any legal liability or responsibility for the accuracy, completeness, or usefulness of any information, apparatus, product, or process disclosed, or represents that its use would not infringe privately owned rights. Reference herein to any specific commercial product, process, or service by trade name, trademark, manufacturer, or otherwise does not necessarily constitute or imply its endorsement, recommendation, or favoring by the United States Government or any agency thereof. The views and opinions of authors expressed herein do not necessarily state or reflect those of the United States Government or any agency thereof. 